\title{
tic\&société
}

Vol. $4, n^{\circ} 1 \mid 2010$

Interactivité et lien social

\section{Le chat est-il (encore) un média interactif?}

Guillaume LATZKO-TOTH

\section{(2) OpenEdition}

\section{Journals}

Édition électronique

URL : http://journals.openedition.org/ticetsociete/751

DOI : 10.4000/ticetsociete.751

Éditeur

Association ARTIC

\section{Référence électronique}

Guillaume LATZKO-TOTH, « Le chat est-il (encore) un média interactif? », tic\&société [En ligne], Vol. 4, n 1 | 2010, mis en ligne le 17 mai 2010, consulté le 19 avril 2019. URL : http://journals.openedition.org/ ticetsociete/751 ; DOI : 10.4000/ticetsociete.751 
tic\&société - 4 (1), 2010

\title{
Le chat est-il (encore) un média interactif?
}

\author{
Guillaume Latzko-Toth \\ LabCMO - Université du Québec à Montréal \\ C.P. 8888, succ. Centre-Ville \\ Montréal (Québec) \\ Canada H3C 3P8
}

latzko-toth.guillaume@uqam.ca

Guillaume Latzko-Toth a récemment obtenu un doctorat en communication à l'Université du Québec à Montréal (UQAM) et poursuit des recherches postdoctorales à l'Université de I'Illinois à Chicago (UIC). II est membre du Laboratoire de communication médiatisée par ordinateur (LabCMO) de I'UQAM ainsi que du Centre interuniversitaire de recherche sur la science et la technologie (CIRST). Ses intérêts de recherche actuels portent sur les dynamiques sociotechniques à l'oeuvre dans le développement des dispositifs de communication médiatisée par ordinateur. II a consacré plusieurs années à l'étude des pratiques de chat sur Internet, tout en menant une réflexion plus générale sur les rapports entre pratiques techniques, pratiques de communication et lien social. 


\title{
Le chat est-il (encore) un média interactif?
}

\begin{abstract}
Résumé
Cet article décrit l'émergence du chat comme dispositif de communication (en analysant la genèse des principaux artefacts) et entreprend de déconstruire l'évidence de son statut de média interactif, d'une part en proposant des " hypothèses fortes " (normatives) sur ce qui fait qu'un média peut être qualifié d'interactif, et d'autre part en montrant que cette forme de communication médiatisée par ordinateur (CMO) s'est articulée de deux façons distinctes. L'une, plutôt conviviale et orientée vers la sociabilité - les " salons » de chat - et l'autre, plus instrumentale et axée sur l'efficacité - les messageries instantanées. L'essor de ces dernières au détriment des espaces de chat "conviviaux " apparaît comme un tournant dans la construction sociale de cette pratique qui reflète la montée de l'individualisme en réseau et nous amène à relativiser l'interactivité du chat en tant que « média social ».
\end{abstract}

Mots-clés : média, interactivité, chat, évolution des médias, usages des médias.

\section{Abstract}

This paper traces the emergence of online chat as a communication device (through analyzing the genenis of main artifacts) and strives to deconstruct the obviousness of its status of interactive media. It does so by first suggesting "strong" (normative) hypotheses on what makes a media "interactive", then by showing that this form of computer-mediated communication (CMC) has been articulated in two different ways. One is rather convivial and sociability-oriented - chat "rooms" - while the other is more instrumental and focused on efficiency - instant messaging systems. The soaring of the latter at the expense of "convivial" chat spaces appears as a turn in the social construction of this practice, reflecting the rise of networked individualism, and inviting us to reassess the interactivity of online chat as a "social media".

Keywords : media, interactivity, online chat, media evolution, media uses. 


\begin{abstract}
Resumen
Este articulo describe el chat como un dispositivo de comunicación (mediante la génesis de los principales artefactos) y pretende deconstruir la evidencia de su estatuto de medio interactivo Para ello, por un lado se proponen "hipótesis fuertes" (normativas) sobre lo que hace que un medio pueda ser calificado de interactivo, y por otro lado, se muestra que esta forma de comunicación mediatizada por ordenador (CMO) se articula de dos maneras distintas. Una, más bien distendida y orientada hacia la sociabilidad -los "salones" de chat- y la otra, más instrumental y centrada en la eficacia -las mensajerías instantáneas. El auge de estas últimas en detrimento de los espacios de chat "distendidos" aparece como un punto de inflexión en la construcción social de esta práctica, que refleja el aumento del individualismo en red y que nos lleva a relativizar la interactividad del chat en tanto que "medio social".
\end{abstract}

Palabras claves : medio, interactividad, chat, evolución de los medios, uso de los medios. 
Le chat est-il (encore) un média interactif ?

\section{Introduction}

La notion d'interactivité, polysémique, demeure une clé pour comprendre l'évolution des dispositifs de communication médiatisée par ordinateur (CMO). Appliquée à l'informatique, la notion d'interactivité renvoie à l'idée d'un dialogue - ou d'une conversation - avec ou à travers la machine (Sénécal, 2006). Face à cette dualité, les analystes des nouveaux médias ont eu tendance à se concentrer sur l'un ou l'autre pôle de l'interaction, contrastant l'interactivité technique avec l'interactivité sociale. Ceux qui se sont attelés à réfléchir globalement sur l'interactivité des médias ont eu tendance à dénoncer comme suspecte la prétention des concepteurs de ces dispositifs à transposer l'interactivité technique en promesses d'interactions sociales (Laulan, 1993; Bardini, 2000b), parlant même de " simulacre d'interaction sociale » (Proulx et Sénécal, 1995).

L'interactivité humain-machine a été conceptualisée par J.C.R. Licklider (1990 [1960]) en tant que " couplage » entre l'humain et l'ordinateur, par le biais d'une conversation continue et en temps réel ${ }^{1}$. Or, Licklider pensait l'interactivité humain-machine en complémentarité avec une autre forme d'interactivité, humain-humain cette fois, ou encore interpersonnelle, c'est-à-dire un « face à face " médiatisé par le dispositif (Licklider et Taylor, 1990 [1968]). Les recherches sur les premiers systèmes de conférence assistée par ordinateur (Vallee et al., 1975; Hiltz et Turoff, 1978) indiquaient déjà que le mode de communication dit synchrone, couramment appelé chat (ou clavardage ${ }^{2}$ ), est celui qui est perçu comme le plus " interactif » par les usagers. Cela se vérifie dans la propension des usagers des TIC à privilégier des dispositifs de communication «instantanée » et des usages sociaux du Web dont la

\footnotetext{
${ }^{1}$ L'une des implémentations du concept est l'informatique à temps partagé fondée sur un modèle dit " conversationnel » en mode texte, devenu un archétype de l'interaction avec l'ordinateur (Bardini, 2000a). Ce modèle a également influencé les premiers systèmes d'information en réseau, et notamment l'offre de services initialement envisagée pour le réseau qui allait devenir Internet (Flichy, 2001), puis pour les services en ligne et télématiques.

${ }^{2}$ Proposé en 1997 par l'Office québécois de la langue française, le terme "clavardage " signifie littéralement: bavardage (sens premier de chat) au clavier. II faut cependant noter avec Pastinelli (2003) l'existence de la pratique du chat vocal, popularisée notamment par des logiciels comme Paltalk. Et que dire d'une application comme Skype, qui combine téléphonie et clavardage ? C'est pourquoi, tant que les usages - aussi bien techniques que linguistiques - ne seront pas stabilisés, nous préférons utiliser le terme anglais chat comme terme générique. Notons qu'en France, le verbe " chatter » est entré dans Le Petit Robert en 2002 ; on voit aussi se propager la version francisée « tchat » (Pierozak, 2003).
} 
temporalité glisse de plus en plus vers le temps réel (cf. le succès de Twitter). Or, généralement considérée comme frivole voire illégitime par les responsables des infrastructures réseau, la pratique du chat, et surtout son implémentation, émane le plus souvent des usagers eux-mêmes ${ }^{3}$. Si bien que l'on peut se demander si le développement des premiers programmes de chat ne correspondrait pas à un processus d'appropriation des infrastructures numériques traduisant une quête d'interactivité, c'est-à-dire une quête de dialogue "vivant " avec et à travers la machine. En ce sens, les dispositifs de chat feraient figure de média interactif par excellence. C'est toutefois la conclusion à laquelle pourraient mener une vision techniciste de l'interactivité et une perception monolithique du phénomène du chat. Cet article commence par décrire l'émergence du chat comme dispositif de communication (en analysant la genèse des artefacts précurseurs) et entreprend ensuite de déconstruire l'évidence de son statut de média interactif, à la lumière d'une définition élargie de l'interactivité d'un média.

\section{L'émergence du chat comme dispositif de communication}

Le terme chat désigne l'usage d'un dispositif informatisé pour échanger des messages écrits en mode synchrone. Est synchrone un dispositif de CMO impliquant l'engagement simultané des interactants dans la communication, à l'instar de la conversation téléphonique, et au contraire de l'échange de courriers électroniques, exemple type de l'interaction médiatisée par ordinateur en mode asynchrone ${ }^{4}$. Notons que l'usage linguistique des internautes tend à réserver le terme chat aux dispositifs principalement basés sur le texte, par opposition à ceux qui privilégient l'interaction vocale ou la visioconférence, ou qui mettent en scène des avatars ${ }^{5}$. Or, si le principe d'une conversation écrite en direct semble bien identifier la pratique du chat, certains paramètres restent indéterminés, notamment les modalités de l'entrée en relation, le format

\footnotetext{
${ }^{3}$ Ces constats sont le fruit d'une recherche doctorale (Latzko-Toth, 2010), dans laquelle nous avons étudié la dynamique et le rôle des usages et des usagers dans l'évolution des dispositifs de chat, dans une perspective sociotechnique.

${ }^{4}$ La typologie synchrone/asynchrone appliquée aux dispositifs de CMO remonte aux premières recherches sur les technologies de communication (voir notamment Vallee, Lipinski et Miller, 1974 ; Vallee et al., 1975; Hiltz et Turoff, 1978; Kerr et Hiltz, 1982) et est encore largement employée aujourd'hui dans l'analyse des dispositifs de CMO (voir Paquin, 2006, p. 71).

${ }^{5} \mathrm{Si}$ l'on peut considérer certains mondes virtuels graphiques primitifs (The Palace) comme des dispositifs centrés sur le chat, on ne peut en dire autant de mondes immersifs tel Second Life, qui offrent une gamme beaucoup plus large de modalités d'interaction.
} 
interactionnel (en dyade ou en groupe), ou encore le degré de synchronie ou " synchronéité » (Vallee et al., 1975). Cette indétermination se traduit dans la diversité des dispositifs dès les origines du chat.

On peut situer l'apparation du chat au début des années 1970, avec le développement sur les ordinateurs à temps partagé des premières plateformes de " conférence assistée par ordinateur " - tels les systèmes EMISARI (1971) et EIES (1974) - ou encore la plateforme d'apprentissage en ligne PLATO. Cette dernière voit naître en 1973 une application " non-officielle », Talkomatic, favorisant la sociabilité informelle entre étudiants au sein de "canaux" préfigurant les bavardoirs modernes (Woolley, 1994). Suit peu après la fonctionnalité "TERM-talk", ajoutée par les développeurs de PLATO à la panoplie des commandes du dispositif (Dear, 2002), et limitée à des conversations en dyade ${ }^{6}$. Considérant les discussions sur Talkomatic comme un usage trivial du dispositif (Woolley, ibid.), les administrateurs de PLATO encourageaient l'utilisation de TERM-talk qu'ils présentaient comme un moyen d'obtenir de l'aide en ligne, préfigurant par là l'usage instrumental de la messagerie instantanée dans les organisations (Licoppe, Proulx et Cudicio, 2010).

Ces deux modalités du chat vont continuer à se différencier par la suite, et s'incarner, une quinzaine d'années plus tard, dans des modèles bien distincts. $\mathrm{Au}$ M.I.T., des informaticiens jettent les bases théoriques du concept de messagerie instantanée (en parlant de " service de notification »), dans le cadre du projet Athena. Cela aboutit au premier système de messagerie instantanée (MI), Zephyr (DellaFera et al., 1988), utilisé d'abord sur le campus du M.I.T., puis dans d'autres universités aux États-Unis et ailleurs. Comme le soulignent DellaFera et al. (ibid.), le principe fondamental qui sous-tend ce dispositif est que le flux de messages est centré sur un usager (via son identifiant), plutôt que sur un lieu (l'adresse physique d'un serveur, un nom de forum...). Le concept est repris par une petite entreprise israélienne, Mirabilis, qui l'adapte à Internet et lance "ICQ » (I seek you) en 1996, lequel servira de modèle aux dispositifs du genre $^{7}$.

Parallèlement, le chat se développe en relation étroite avec les services en ligne et les jeux en réseau. En 1980, le service en ligne CompuServe lance l'application CB Simulator, basée sur la métaphore de la Citizens Band (CB) et

\footnotetext{
${ }^{6}$ II s'agissait d'une option intégrée dans l'interface permettant à un usager de contacter un autre usager tout en poursuivant l'utilisation d'une application en cours, et de dialoguer dans une zone de deux lignes réservée à cet effet au bas de l'écran.

${ }^{7}$ D'ailleurs, Mirabilis déposera une demande de brevet aux États-Unis, qui, bien que controversée, sera accordée en 2002 à AOL, qui avait fait l'acquisition de la start-up israélienne en 1998.
} 
qui deviendra son service phare auprès de ses usagers grand public. Fondé en 1983, le service en ligne PlayNet offrait une collection de jeux en réseau, parmi lesquels un jeu d'échecs dont l'originalité était de permettre aux joueurs de chatter entre eux tout en jouant; ils pouvaient également s'envoyer des courriers électroniques et des messages instantanés («On Line Messages ») ${ }^{8}$. Le service fut racheté en 1985 par Control Video Corporation, qui le rebaptisa QuantumLink (Q-Link). L'application phare de Q-Link fut un jeu massivement multijoueurs, un univers graphique persistant appelé Habitat et créé par Lucasfilm en 1986. Conçu pour accueillir jusqu'à 20000 utilisateurs simultanément (Morningstar et Farmer, 1991), ce jeu, précurseur de The Palace et de Second Life, faisait une large part à la sociabilité entre les utilisateurs. Ceux-ci étaient représentés par des avatars en deux dimensions et leurs conversations apparaissaient dans des phylactères (Yakal, 1986). Mais il s'agissait d'un projet-pilote, et l'expérience fut interrompue en 1988. En 1991, l'opérateur de $\mathrm{Q}$-Link changea de nom pour America Online (AOL).

Parallèlement, on voit fleurir une pléthore de systèmes de chat favorisant des rencontres entre usagers ne se connaissant pas et la sociabilité en groupe, dont I'IRC (Internet Relay Chat), créé en 1988 et inspiré d'un dispositif similaire développé sur le réseau BITNET. Ces espaces de chat ont des racines communes avec les premiers jeux en ligne multiutilisateurs persistants, et notamment les MUD, qui à leurs débuts (1978), et selon l'un de leurs inventeurs, Richard Bartle, n'étaient « rien de plus qu'une série de lieux interconnectés où l'on pouvait se déplacer et chatter" (Bartle, 1990). Un peu plus tard, l'application TinyMUD (1989) marque une bifurcation dans l'histoire des mondes virtuels textuels, entre d'un côté des applications résolument ludiques axées sur la rivalité entre joueurs et la fantaisie (quêtes, monstres, etc.) et, de l'autre, des univers où l'accent est mis sur la sociabilité entre les usagers, des MUD dépourvus de l'aspect ludique et dont seules les fonctions de chat ont été conservées : les «talkers ». ${ }^{9}$

Cette conception du chat en tant qu'espace d'interactions sociales suppose l'existence d'un lieu partagé relativement persistant - évoqué par diverses métaphores : salon, canal, forum - au sein duquel les usagers se rassemblent et par le biais duquel il leur est possible de découvrir d'autres usagers du dispositif et de tisser des liens sociaux avec eux. Comme nous l'avons constaté

${ }^{8}$ Voir http://en.wikipedia.org/wiki/PlayNET

${ }^{9}$ On retrouve cette bifurcation dans les mondes virtuels graphiques d'aujourd'hui. Rémi Sussan (2010) reprend la typologie proposée par Roger Caillois (Caillois, 1967, p. 48), qui distingue les jeux fondés sur la paidia (libre expression, absence de règles) de ceux relevant du ludus (règles rigides, but à atteindre). II note que World of Warcraft s'inscrit résolument dans le ludus, tandis que Second Life s'incrirait dans la paidia. 
dans nos recherches, ces interactions sont souvent stimulées par l'horizon de la rencontre en face à face ${ }^{10}$.

Ce rapide survol des origines du chat nous permet donc de constater l'émergence concomitante de deux modes d'articulation distincts du chat, l'un, plutôt instrumental, égocentré et axé sur l'efficacité - dont le dispositif type est la messagerie instantanée - et l'autre, plus convivial ${ }^{11}$ et orienté vers la sociabilité au sein d'un lieu virtuel - sur le modèle du forum électronique. L'IRC apparaît comme l'archétype du dispositif de chat conçu comme espace social partagé.

\section{L'IRC, un média « participatif »}

Le phénomène des "tribus IRC »- des collectifs locaux de chatteurs se formant en ligne mais évoluant vers la «multimodalité » des interactions et la production de lien social ${ }^{12}$ - contribue pour beaucoup à faire de I'IRC un média "participatif». Mais si nous avons vu dans ce dispositif de chat un cas " exemplaire » de dispositif sociotechnique (Latzko-Toth, 2000), c'est plus encore pour la façon dont il a été co-construit par l'ensemble des acteurs concernés par son développement et son utilisation.

Le fait est rarement souligné, mais l'IRC est l'un des plus anciens projets de logiciel libre encore en développement. En effet, après avoir créé le noyau du dispositif seul - à l'instar de son compatriote Linus Torvalds pour Linux, Jarkko Oikarinen a décidé en 1990 de placer le code source du logiciel serveur - pièce maîtresse du dispositif - sous la protection de la licence $\mathrm{GPL}^{13}$, afin de susciter la collaboration d'un maximum de contributeurs (Oikarinen, 2004) :

\footnotetext{
${ }^{10}$ Paradoxalement, la pratique collective de ce système de communication "déterritorialisée " a abouti à la formation de "tribus IRC », des communautés locales d'usagers ancrées dans leur territoire géographique d'appartenance, et dont le mode d'«être-ensemble " oscille entre la communication médiatisée et le face à face (Latzko-Toth, 1998). Sur la rencontre comme horizon d'attente, voir aussi Pastinelli, 2007, p. 215.

${ }^{11}$ Nous entendons ici la notion de convivialité au sens d'lllich (1973), c'est-à-dire la condition d'un corps social qui « cesse d'être dominé par les outils qu'il crée » (Bolle De Bal, 1985, p. 134). Bolle De Bal l'associe à un idéal communautaire (ibid.), tandis que selon Jouët (1992), le double sens en informatique du mot convivialité renvoie à l'espoir que l'appropriation de l'ordinateur par les noninformaticiens se traduise dans la société « par un projet de convivialité [consistant en un] tissage de nouveaux liens entre les usagers » (p. 182).

${ }^{12}$ Dans le cas que nous avons étudié (Latzko-Toth, ibid.), cette socialité a eu plusieurs retombées " concrètes ", c'est-à-dire des formes institutionnalisées et pérennes: ligue d'improvisation théâtrale, entreprise (un fournisseur d'accès Internet), etc. Ce sont là de bons indicateurs de création de lien social, mais cela n'en représente que la partie « émergée ».

${ }^{13}$ La General Public Licence du Projet GNU - ou " copyleft » - élaborée en 1989 par Richard Stallman est à ce jour la plus répandue des licences visant à définir le statut juridique d'un logiciel
} 


\section{Guillaume LATZKO-TOTH}

Céder le contrôle et donner aux autres le pouvoir de travailler, d'apprendre et de développer un logiciel permet de nouvelles innovations. [...] IRC a commencé comme un exercice de programmation durant un stage d'été. De simple "hack», c'est devenu un projet de développement de logiciel auquel des centaines de personnes ont participé, et qui est devenu un environnement mondial au sein duquel maintenant des dizaines de milliers de personnes passent du temps ensemble. (p. vii, trad. libre) ${ }^{14}$

Le développement collaboratif du logiciel serveur n'est pas le seul aspect du processus de co-construction de l'IRC en tant que dispositif. L'analyse que nous avons menée de l'évolution des deux premiers grands réseaux IRC - EFnet et Undernet - nous a permis de mettre en évidence le fait que les usagers se sont mobilisés ou ont été mobilisés dès le début dans la construction du dispositif (Latzko-Toth, 2010). Par exemple, en inventant des solutions pour pallier les carences de la "donne » technique initiale, comme le robot-gardien qui permet de garder " par délégation » le contrôle sur un canal en l'absence d'opérateurs légitimes, ou encore le robot «NickServ » qui signale qu'un pseudonyme est déjà utilisé sur une base régulière, alors que le protocole originel ne prévoit ni la protection des canaux, ni l'enregistrement des pseudonymes. En quelque sorte, les usagers "ordinaires " s'invitent ainsi comme co-concepteurs de l'infrastructure logicielle de I'IRC, distribuée en une myriade de robots et autres " services" non officiels. Bien qu'elles soient souvent sujettes à controverse, ces contributions de l'usager participent au processus de construction et de différenciation des réseaux IRC, autour de " philosophies " du chat différentes qui caractérisent des communautés de pratique du chat elles-même distinctes.

Dans le cas du réseau Undernet, qui devait se positionner par rapport à son prédécesseur EFnet, ses fondateurs ont opté pour l'enrôlement institutionnel des usagers en créant des comités de gestion du réseau auxquels ils sont intégrés ${ }^{15}$. Mentionnons également la mobilisation des usagers pour " sauver » Undernet lorsqu'il a été la cible d'attaques massives en janvier 2001. En effet,

libre. Très tôt, Jarkko Oikarinen s'est montré ouvert à ce que le code d'IRC soit libre, à condition que sa paternité demeure reconnue.

${ }^{14}$ Précisons que contrairement à Linus Torvalds avec le noyau de Linux, Jarkko Oikarinen s'est retiré très tôt du rôle de coordonnateur du développement d'IRC.

${ }^{15}$ Notamment, le "comité des usagers " (User Committee) et le "comité du service de canaux " (Channel Service Committee), ce dernier visant à réduire les inégalités dans l'appropriation du dispositif en permettant à des non-informaticiens de disposer des mêmes outils pour administrer un canal que ceux qui ont les ressources et les connaissances pour mettre en œuvre leur propre automate. 
Le chat est-il (encore) un média interactif ?

deux mois durant, les usagers durent réapprendre à s'organiser et à se responsabiliser comme avant l'âge des robots, les opérateurs humains se relayant sur les canaux pour assurer la garde de leur canal vingt-quatre heures sur vingt-quatre en se tenant prêts à faire face aux tentatives de prise de contrôle hostile des canaux par les script kiddies ${ }^{16}$ (Undernet User Committee, 2003). Comme le notent Long et Allen (2001), cette situation chaotique aboutit au résultat paradoxal que les usagers furent revalorisés, et que le lien social entre eux ainsi que leur sentiment d'appartenance à leur canal et au réseau Undernet, s'en trouvèrent renforcés.

Une autre caractéristique intéressante de I'IRC est d'être le seul service Internet majeur dont le fonctionnement nécessite la vigilance constante et l'intervention continue d'opérateurs humains pour fonctionner correctement ${ }^{17}$. La maintenance technique du réseau est assurée en temps réel par les "IRCops" (opérateurs IRC), lesquels tirent un grand prestige social de cette responsabilité qui consacre le haut niveau d'habileté technique de son titulaire ${ }^{18}$. Quant aux espaces de discussion proprement dits -les canaux - ils créés et administrés par les usagers eux-mêmes. En constatant les liens forts qui se sont tissés entre les acteurs du développement de I'IRC au fil des années, nous en arrivons à nous demander si la construction même du dispositif, au-delà de la valeur d'usage qu'elle procure, n'est pas un prétexte à la sociabilité, par le biais de la constitution d'une communauté de pratique (Wenger, 2000) qui donne un sens à l'engagement des acteurs.

\section{3. À propos de l'interactivité des médias interactifs}

Nous avons fait valoir en introduction que la possibilité de converser en temps réel à travers le dispositif contribuait à rendre celui-ci interactif. Mais qu'est-ce qu'un média interactif? La difficulté, lorsque l'on entreprend une réflexion critique sur l'interactivité d'un dispositif, est de disposer d'une définition claire et communément partagée de la notion d'interactivité. Malheureusement, comme pour bien des notions intéressant les chercheurs en communication, il

\footnotetext{
${ }^{16}$ Terme condescendant du jargon hacker désignant des pirates en herbe ou cybervandales qui se contentent d'utiliser de petits programmes ou bouts de code (scripts) - dont ils ne sont généralement pas les auteurs - et ce, dans le seul but de provoquer le plus de dommages possible.

${ }^{17}$ En ce sens, l'IRC est un dispositif interactif au sens proposé par Jean-Marie Balpe, qui l'oppose à « automatique » (Balpe, 1990, cité dans Julia, 2003, p. 205).

${ }^{18}$ L'accession au statut d'opérateur constitue donc une motivation forte pour plusieurs usagers à s'investir dans l'apprentissage des arcanes du dispositif, tant dans ses aspects techniques que sociaux - aide aux usagers débutants, participation aux forums de dévéloppement des réseaux (listes de discussion, groupes Usenet...).
} 


\section{Guillaume LATZKO-TOTH}

s'agit d'un " mot-valise " dont la détermination du sens est elle-même l'enjeu d'âpres débats théoriques et épistémologiques. II y a quelques années, alors que nous cherchions une définition concise et opératoire à l'intention d'un public étudiant, nous sommes tombé sur la définition du Dictionnaire des arts médiatiques réalisé par le Groupe de recherche en arts médiatiques de I'Université du Québec à Montréal :

Propriété des médias, des programmes et des systèmes liés de façon plus ou moins constitutive à un ordinateur de pouvoir entretenir un dialogue plus ou moins poussé avec l'utilisateur. Les hypertextes et les hypermédias [...] sont des entités informatiques fondamentalement interactives, qui nécessitent constamment [...] les réponses des utilisateurs aux choix qu'ils leur offrent [...]. Ces réponses relèvent du processus de navigation des utilisateurs dans ces programmes et ces systèmes. [...] Dans le domaine des arts, possibilité pour le spectateur de participer à la réalisation de l'oeuvre. [...] (GRAM, 1996; termes soulignés par nous)

Cette définition nous a paru synthétiser les dimensions essentielles d'un média interactif : dialogue, navigation, participation. Cette dernière dimension, bien que rattachée aux oeuvres artistiques dans la définition ci-dessus, est fréquemment mise de l'avant dans les discours sur l'interactivité des nouveaux médias ${ }^{19}$. Dans un média interactif, l'usager peut donc :

- Dialoguer (« interagir ») avec le dispositif-contenu ;

- Choisir son parcours (i.e. " naviguer ») dans le dispositif-contenu ;

- Participer (contribuer) au dispositif-contenu.

Nous employons l'expression " dispositif-contenu » pour exprimer les deux facettes du média interactif : le dispositif technique et le message qu'il véhicule. II faut concevoir l'interactivité comme une qualité plus ou moins prononcée, et comprendre les indicateurs ci-dessus comme des "palliers" d'une gradation, d'une échelle d'interactivité ${ }^{20}$. Dans le même ordre d'idées, le trait d'union représente un « ou » inclusif, au sens où il se peut que l'usager puisse intervenir à la fois sur le dispositif et sur le contenu, ou seulement sur le contenu, mais pas sur le dispositif, et vice-versa.

Sur cette base, nous pouvons d'ores et déjà noter que l'interactivité d'un média ne va pas de soi et qu'elle est au contraire toujours problématique. Par exemple, on a longtemps considéré le Web comme un média interactif " par

\footnotetext{
${ }^{19}$ Voir par exemple Paquin (2006, p. 76-77).

${ }^{20}$ Pour un modèle de l'interactivité qui s'efforce d'opérationnaliser cette gradation, voir McMillan (2002).
} 
définition ", puisque reposant sur le principe de la navigation hypertexte, qui subsume le dialogue avec la machine. Mais qu'en est-il de la dimension participative, au-delà d'une conception de la participation restreinte à la possibilité de choisir son parcours ? Ce que l'on pourrait appeler le «Web de première génération " a eu tendance à reproduire le modèle des médias de masse. Certes, tout un chacun pouvait créer son propre site, mais lorsqu'on regardait quels sites les usagers consultaient le plus, on constatait que c'étaient toujours les dix mêmes sites, à peu près, lesquels étaient souvent dans le giron de grands groupes médiatiques (George, 2008). En revanche, ce que l'on a désigné par l'expression «Web 2.0 » (O'Reilly, 2005), c'est l'invention d'un média réellement nouveau ${ }^{21}$, une façon de «faire du Web » qui repose sur la participation massive des usagers, devenus des contributeurs (Proulx, 2009), des "produtilisateurs" (Bruns, 2008); bref, sur le harnachement de ce qu'Olivier Blondeau appelle "l'expressivisme » des usagers (Blondeau et Allard, 2007).

Mais le Web nouveau n'est pas seulement " participatif »; il est de plus en plus fréquemment qualifié de "social » (Girgensohn et Lee, 2002 ; Millerand, Proulx et Rueff, 2010). C'est cette socialité dans le dispositif qui semble la caractéristique la plus prégnante du Web contemporain. Autrement dit, son interactivité au sens d'interactivité sociale, c'est-à-dire sa capacité à susciter des interactions entre ses usagers. II ne s'agit plus seulement que l'usager participe au dispositif (ou au média), mais que le dispositif (ou le média) fasse participer les usagers à une dynamique collective (voir Blondeau et Allard, ibid.).

Nous prenons ainsi le parti d'une conception humaniste, et non techniciste, de l'interaction au sein du dispositif interactif ${ }^{22}$. En effet, le triptyque dialoguenavigation-participation est souvent traduit en termes de qualité de l'expérience sensorielle "immersive " dans le média. Vu sous cet angle, le "média » interactif ultime serait le face à face (par définition), et juste après viendrait la réalité virtuelle telle qu'elle est définie par l'inventeur du concept, Jaron Lanier (1988), c'est-à-dire une simulation pleinement fonctionnelle du réel par le truchement d'une interface sollicitant tous les canaux sensoriels. Les premiers psychosociologues qui ont théorisé la communication médiatisée par ordinateur

\footnotetext{
${ }^{21}$ Cette tendance des nouveaux médias à «imiter " un média plus ancien à ses débuts ou à reproduire des formats existants est un phénomène connu, théorisé notamment par Meyrowitz (1985) et Ong (1982). La télévision, par exemple, a mis du temps à se démarquer de la radio et à trouver sa " personnalité » propre.

${ }^{22}$ II ne s'agit pas ici d'exclure la dimension conversationnelle humain-machine. Celle-ci est très présente, par exemple, dans le cas de l'IRC où l'usager adresse, dans la même zone de saisie, aussi bien des messages au serveur qu'aux autres usagers, parmi lesquels des usagers " simulés " (robots). Il s'agit plutôt d'inclure, dans la conversation interactive, des actants aussi bien humains que machiniques.
} 
ont ainsi construit des variables technicistes telles que la " présence sociale » et la "largeur de bande du média » fondées sur ce présupposé que plus il y a de " canaux » mobilisés et plus « riche » est le signal, plus l'expérience médiatique est interactive (Short, Williams et Christie, 1976 ; Daft et Lengel, 1984, 1986). De ce point de vue, l'interactivité d'un dispositif de chat semble bien pauvre, puisque le seul "canal » mobilisé est le texte (Rintel et Pittam, 1997). Or, des recherches récentes sur les usages des dispositifs de CMO ont fortement remis en cause ces inférences teintées de déterminisme technique, montrant notamment que les interactions via les médias "texte" pouvaient être aussi riches, voire plus élaborées que celles impliquant la voix et l'image (Watt, Lea et Spears, 2002). D'ailleurs, l'observation des usages d'Internet confirme la persistance de la $\mathrm{CMO}$ en mode texte, en dépit des prédictions et des possibilités techniques offertes par le déploiement de réseaux multimédia à haut débit (Herring, 2004). Cela tend à démontrer qu'un dispositif de communication médiatisée par ordinateur n'est pas nécessairement, intrinsèquement, un média interactif. II faudrait plutôt considérer l'interactivité comme une qualité émergente d'un dispositif médiatique - notamment à travers ses usages - et non comme le produit de caractéristiques intrinsèques.

\section{Une mutation dans les usages du chat?}

Nous l'avons évoqué plus haut: on peut identifier, dès les origines du chat, deux modèles ou "formats interactionnels » sous-jacents aux dispositifs qui lui sont associés. Or nous constatons le déclin, durant la dernière décennie, d'un modèle du chat fondé sur la métaphore du café, du salon - et parfois de la rue pendant que semble s'imposer un modèle de communication filtrée dont l'emblème pourrait être le réseau d'» ego » en étoile, et qui caractérise les messageries instantanées (MI) fondées sur la gestion d'une «liste de contacts ". Tandis que la fréquentation de salons de chat stagne voire tend à décliner $^{23}$ (Pastinelli, 2007), les MI jouissent d'une popularité grandissante, tant auprès du grand public que des entreprises (Herring, 2004; Boboc, 2005 ; Licoppe, Proulx et Cudicio, 2010) ${ }^{24}$. Cette évolution semble être en phase avec

\footnotetext{
${ }^{23}$ Par exemple, le seul réseau IRC majeur en croissance actuellement est Freenode, voué à la communauté du logiciel libre (source : www.netsplit.de, février 2010). Tous les autres sont en déclin depuis 2004-2005. Mais cela ne se fait pas nécessairement au profit de dispositifs plus sophistiqués, puisque Second Life connaît aussi un déclin de sa fréquentation (voir Hansen, 2009).

${ }^{24}$ Une récente étude menée auprès d'un échantillon représentatif des personnes âgées de plus de 14 ans indique que $26 \%$ des internautes britanniques fréquentent les salons de chat tandis que $56 \%$ pratiquent la MI (Gennaro et Dutton, 2007). Une étude canadienne appuie cette tendance en indiquant que $97 \%$ des étudiants utilisent la MI (Quan-Haase, 2008).
} 
une tendance lourde dans nos sociétés, l'adoption d'un mode prépondérant de sociabilité que Castells (2002) appelle "l'individualisme en réseau » :

Après l'hégémonie des relations primaires (les familles, les communautés), puis secondaires (les associations), nous voici dotés d'un nouveau système dominant qui paraît construit sur les relations tertiaires, pourrait-on dire - ce que Wellman appelle les "communautés personnalisées » : les réseaux centrés autour du moi. On assiste, en fait, à une véritable privatisation de la sociabilité. (Castells, 2002, p. 161)

Castells précise que " l'individualisme en réseau est une structure sociale, et non une juxtaposition d'individus isolés » (p. 164), et ajoute que ce ne sont pas les nouveaux médias réticulaires qui engendrent cette forme sociale, mais qu'ils en sont plutôt les révélateurs.

En particulier, nous soulignerons deux aspects de cette tendance :

- La diminution de l'offre d'espaces de chat en groupe et de la participation des usagers à la construction et à l'administration de ces espaces ;

- La tendance de l'internaute au repli dans une sphère de communication privée, centrée sur lui et au sein de laquelle il agit en monarque absolu, mais aussi en consommateur, l'Autre - le « contact » présélectionné - devenant une ressource exploitée au gré des envies et des besoins ${ }^{25}$.

La rencontre fortuite, la confrontation à l'altérité, la gestion de la conflictualité et la conciliation des différences au sein des collectifs en ligne, bref, toutes les aspérités de l'être-semble et de la sociabilité sont en quelque sorte gommées des nouveaux dispositifs de chat égocentrés tels que Live Messenger, Google Talk, Skype ou encore le module de chat intégré au site Facebook. L'entreprise Microsoft a bien perçu l'air du temps en décidant, en 2006, de fermer définitivement ses espaces de clavardage publics (MSN Chat), reposant largement sur le protocole IRC ${ }^{26}$. Le motif officiel invoqué par Microsoft était la

${ }^{25}$ Dans le même ordre d'idées, Eva Illouz (2006) note la pénétration du modèle capitaliste dans la sphère privée, et ce qu'elle appelle une mise en marché du soi, notamment dans les interactions sur les sites de rencontre (chap. 3 ), où elle observe une rationalisation des interactions et une réification de soi et de l'autre. Ce rapprochement nous a été suggéré par Julien Rueff.

${ }^{26}$ Notons qu'après avoir consacré en vain d'importants efforts à faire adopter par l'IETF (Internet Engineering Task Force - instance en charge d'établir les standards d'Internet) un standard soidisant plus robuste que le "vieux » protocole IRC - IRCX, pour IRC eXtensions - le géant du logiciel a abandonné ce projet au profit d'un protocole de messagerie instantanée, Messenger. 


\section{Guillaume LATZKO-TOTH}

non-rentabilité du service. Le mouvement avait été amorcé trois ans plus tôt, en septembre 2003, lorsque Microsoft avait fermé ses salons de clavardage en Europe et en Asie, officiellement pour protéger les mineurs des prédateurs sexuels, les salons continuant à être accessibles dans les pays où leur accès était tarifé, comme aux États-Unis et au Canada ${ }^{27}$. On peut se demander si le virage opéré par Microsoft n'est pas lié à la plus grande " marchandisabilité » de la messagerie instantanée propriétaire et ce, dans la mesure où elle impose un client spécifique qui expose l'usager à des bandeaux publicitaires dont on peut mesurer précisément l'audience. Cependant, le fait de ne pas avoir à gérer les problèmes sociotechniques parfois épineux émergeant dans les espaces de conversation publics constitue probablement une autre motivation pouvant expliquer la décision de Microsoft, qui a d'ailleurs été imitée peu après par Yahoo! ${ }^{28}$. Plus récemment, c'est Skype qui a mis fin abruptement à ses salons de chat publics (Courtney, 2009).

L'ère des vastes "cités électroniques" (Reid, 1991) où convergeaient naguère les internautes pour dialoguer en temps réel est-elle révolue ? On constate une fragmentation et une spécialisation croissantes des espaces de CMO synchrone ${ }^{29}$. De plus en plus, les dispositifs de chat sont subordonnés à des sites de rencontre et de "réseautage social » où la tendance est au filtrage de la communication. Parallèlement, on assiste à l'essor d'un mode de sociabilité où la coprésence prime sur l'interaction réelle (Quan-Haase et Collins, 2008), et où l'être-ensemble prend l'aspect d'une " solitude collective » (Ducheneaut et Yee, 2008).

\footnotetext{
${ }^{27}$ Voir le communiqué officiel de Microsoft: "Protecting children online », http://www.microsoft.com/about/corporatecitizenship/citizenship/internetsafety/protectingchildren.ms px

Yahoo! a décidé, en juin 2005, la fermeture de tous les salons créés par les usagers (Sullivan, 2005). Motif invoqué : l'effroi des annonceurs à l'idée que leur image puisse être associée à des discussions publiques contraires aux bonnes moeurs.

${ }^{29}$ À titre d'exemple, on recense aujourd'hui près de 5500 réseaux IRC (source: SearchIRC.com, février 2010).
} 
Le chat est-il (encore) un média interactif ?

\section{Conclusion}

Alors que l'idée d'un usager non seulement acteur, mais aussi contributeur des dispositifs techniques est de plus en plus mise de l'avant aujourd'hui (Bruns, 2008 ; Proulx, 2009), nous constatons que la nature de cette contribution est souvent limitée au contenu. Ainsi, la place des usagers ordinaires dans le façonnement d'un dispositif comme Facebook est marginale : consultation sur la charte d'utilisation des données, participation bénévole à la traduction de l'interface pour la rendre multilingue... Le cas de l'IRC est intéressant en ce sens qu'il donne à voir la contribution des usagers à la structure même du dispositif. Toutefois, l'IRC ne représente plus le modèle dominant du chat tel qu'il se pratique aujourd'hui. Le déplacement que l'on observe, dans les pratiques de chat, des dispositifs de sociabilité électronique centrés sur un lieu virtuel (salons) aux dispositifs centrés sur l'individu (messageries instantanées) tendrait à accréditer la thèse de Gérard Dubey (2001) qui affirme que « non seulement les technologies virtuelles sont impuissantes à produire à elles seules du lien social [...] mais l'efficacité qu'on leur attribue [...] dépend presque entièrement de la préexistence de celui-ci » (p. 190). L'interactivité du chat, avec la conception élargie que nous avons proposée (incorporant les dimensions sociale et participative), serait ainsi galvaudée puisque on ne pourrait véritablement parler de capacité du dispositif sociotechnique à susciter des interactions en dehors d'un cercle étroit, filtré, prédéfini - autrement dit sociales et non intimes; bref, à encourager la participation des usagers à des dynamiques collectives.

A contrario, l'usage des messageries instantanées s'inscrit dans un processus de "numérisation » du réseau social personnel et tend à transformer ce dernier en ressource pour l'individu, en capital social mobilisable en réponse à divers problèmes auxquels l'individu peut être confronté dans sa vie quotidienne (Pénard et Poussing, 2006 ; Best et Krueger, 2006). À l'aube du $\mathrm{XXI}^{\mathrm{e}}$ siècle, l'instrumentation de nos relations sociales et affectives, de plus en plus complexe et diversifiée, nous oblige individuellement et collectivement à nous interroger sur la nature même du lien que nous tissons avec les autres et sur notre façon de gérer ce lien dans un contexte de rationalisation et d'objectivation croissante - voire d'instrumentalisation - des relations interpersonnelles par la médiation des dispositifs techniques. Questionner l'interactivité des dispositifs comme une propriété émergente et non comme une donnée de leur fonctionnement participe d'une telle démarche. 


\section{Références bibliographiques}

BALPE J.-P., 1990, Hyperdocuments, hypertextes, hypermédias, Paris, Eyrolles.

BARDINI T., 2000a, Bootstrapping: Douglas Engelbart, Coevolution, and the Origins of Personal Computing, Stanford, Stanford University Press.

BARDINI T., 2000b, "Les promesses de la révolution virtuelle: genèse de l'informatique personnelle, 1968-1973 », Sociologie et sociétés, vol.32, n², pp.57-72.

BARTLE R. A., 1990, "Early MUD History », <http://www.mud.co. uk/richard/mudhist.htm>, dernière consultation le 3 mars 2010.

BEST S. J. et B. S. KRUEGER, 2006, "Online Interactions and Social Capital: Distinguishing Between New and Existing Ties», Social Science Computer Review, vol.24, n4, pp.395-410.

BLONDEAU O. et L. ALLARD, 2007, Devenir média. L'activisme sur Internet, entre défection et expérimentation, Paris, Éditions Amsterdam.

BOBOC A., 2005, « Le point sur la messagerie instantanée. Solutions grand public (IM) et solutions d'entreprise (EIM) », Réseaux, vol.2005/6, n¹34, pp.223-261.

BOLLE DE BAL M., 1985, La tentation communautaire. Les paradoxes de la reliance et de la contre-culture, Bruxelles, Éd. de l'Université de Bruxelles.

BRUNS A., 2008, Blogs, Wikipedia, Second Life, and Beyond: From Production to Produsage, New York, Peter Lang.

CAILLOIS R., 1967, Les jeux et les hommes. Le masque et le vertige éd. revue et augmentée, Coll. « Idées », Paris, Gallimard.

CASTELLS M., 2002, La Galaxie Internet, Paris, Fayard.

COURTNEY J., 2009, "Skype for Windows 4.0 Goes Gold; Improved UI, Audio and Video Performance ", Skype Journal, <http://skypejournal.com/2009/02/skype-for-windows-40-goes-goldimproved.html>, dernière consultation le 28 janvier 2010.

DAFT R. L. et R. H. LENGEL, 1986, «Organizational Information Requirements, Media Richness and Structural Design », Management Science, vol.32, n5, pp.554-571. 
Le chat est-il (encore) un média interactif ?

DAFT R. L. et R. H. LENGEL, 1984, «Information Richness: A New Approach to Managerial Behavior and Organizational Design », dans L. L. CUMMINGS et B. M. STAW (dir), Research in Organizational Behavior, Homewood, JAI Press, pp.191-233.

DEAR B., 2002, «TERM-talk: PLATO's Instant Messaging ». En ligne. <http://www.platopeople.com/termtalk.html>, dernière consultation le 3 mars 2010.

DELLAFERA C. A., M. W. EICHIN, R. S. FRENCH, D. C. JEDLINSKY, J. T. KOHL et W. E. SOMMERFELD, 1988, "The Zephyr Notification Service ", dans Proceedings of Winter 1988 Usenix Conference, $<$ http://www.rfrench.org/papers/ usenix.pdf>, dernière consultation le 3 mars 2010.

DUBEY G., 2001, Le lien social à l'ère du virtuel, Coll. "La politique éclatée ", Paris, PUF.

DUCHENEAUT N. et N. YEE, 2008, «Collective solitude and social networks in World of Warcraft », dans C. T. ROMM et K. SETZEKORN (dir), Social Networking Communities and E-dating Services: Concepts and Implications, Hershey, IGI Global, pp.78-100.

FLICHY P., 2001, L'imaginaire d'Internet, Paris, La Découverte.

GENNARO C. D. et W. H. DUTTON, 2007, «Reconfiguring Friendships: Social Relationships and the Internet », Information, Communication \& Society, vol.10, n5, pp.591-618.

GEORGE É., 2008, "La concentration de la propriété des entreprises médiatiques à l'heure du Web », dans M. MEIMARIS et D. GOUSCOS (dir), Actes du 3e colloque EUTIC (Enjeux et Usages des TIC), Athènes, Gutenberg, pp.42-51.

GIRGENSOHN A. et A. LEE, 2002, "Making web sites be places for social interaction », Proceedings of the 2002 ACM conference on Computer supported cooperative work, New Orleans,

GRAM, 1996, Dictionnaire des arts médiatiques. Montréal, Groupe de recherche en arts médiatiques, UQAM, <http://www.dictionnairegram.org/>, dernière consultation le 17 février 2010.

HANSEN L., 2009, "What happened to Second Life? ». BBC News Magazine, 20/11/2009, <http://news.bbc.co.uk/2/ hi/uk_news/magazine/8367957.stm>, dernière consultation le 20 novembre 2009. 
HERRING S. C., 2004, "Slouching Toward the Ordinary: Current Trends in Computer-Mediated Communication », New Media \& Society, vol.6, n¹, pp.2636.

HILTZ S. R. et M. TUROFF, 1978, The Network Nation : Human Communication via Computer, Reading ; Don Mills, Addison-Wesley.

ILLICH I., 1973, La convivialité, Paris, Seuil.

ILLOUZ E., 2006, Les sentiments du capitalisme, Paris, Seuil.

JOUËT J., 1992, "Relecture de la société de l'information », dans P. CHAMBAT (dir), Communication et lien social, Paris, Descartes, pp.177-190.

JULIA J.-T., 2003, « Interactivité, modes d'emploi. Réflexions préliminaires à la notion de document interactif », Documentaliste-Sciences de l'information, vol.40, n²003/3, pp.204-212.

KERR E. B. et S. R. HILTZ, 1982, Computer-mediated communication systems : status and evaluation, New York ; Toronto, Academic Press.

LANIER J., 1988, "A Vintage Virtual Reality Interview » (Interview with Adam Heilbrun), Whole Earth Review, <http://www.jaronlanier.com/vrint.html>, dernière consultation le 4 novembre 2009.

LATZKO-TOTH G., 2010, « La co-construction d'un dispositif sociotechnique de communication : le cas de l'Internet Relay Chat ", thèse de doctorat en communication, Université du Québec à Montréal.

LATZKO-TOTH G., 2000, "L'Internet Relay Chat : un cas exemplaire de dispositif sociotechnique ", COMMposite, v2000.1, <http://commposite.org/index.php/revue/article/view/ 91/68>, dernière consultation le 3 mars 2010.

LATZKO-TOTH G., 1998, "À la rencontre des tribus IRC: le cas d'une communauté d'usagers québécois de I'Internet Relay Chat ", mémoire de maîtrise en communication, Université du Québec à Montréal, <http://archivesic.ccsd.cnrs.fr/docs/00/46/ 12/32/PDF/LatzkoToth_1998_tribus_IRC.pdf>, dernière consultation le 4 mars 2010.

LAULAN A.-M., 1993, "Interactivité et lien social », Les Cahiers du CIRCAV, $\mathrm{n}^{\circ} 3$ : « Interactivité ».

LICKLIDER J. C. R., 1990 [1960], "Man-Computer Symbiosis », SRC Research Report, $n^{\circ} 61$, pp.1-19, <ftp://gatekeeper.research.compaq.com/pub/DEC/SRC/research-reports/SRC- 
061.pdf> (Initialement publié dans la revue IRE Transactions on Human Factors in Electronics, mars 1960), dernière consultation le 3 mars 2010.

LICKLIDER J. C. R. et R. W. TAYLOR, 1990 [1968], «The Computer as a Communication Device ", SRC Research Report, $\mathrm{n}^{\circ} 61$, pp.21-41, <ftp://gatekeeper.research.compaq. com/pub/DEC/SRC/research-reports/SRC061.pdf> (Initiale-ment publié dans la revue Science and Technology, avril 1968), dernière consultation le 3 mars 2010.

LICOPPE C., S. PROULX et R. CUDICIO, 2010, "Contribution et coopération à distance via l'usage de messageries instantanées en entreprise », dans F. MILLERAND, S. PROULX et J. RUEFF (dir), Web social, mutation de la communication, Québec, Presses de l'Université du Québec, pp.233-251.

LONG J. et M. ALLEN, 2001, "Hacking the Undernet: Libertarian limits; commercial containment », Australian Journal of Communication, vol.28, $\mathrm{n}^{\circ} 3$, pp.37-44.

MCMILLAN S. J., 2002, "A four-part model of cyber-interactivity: Some cyber-places are more interactive than others ", New Media Society, vol.4, $n^{\circ} 2$, pp.271-291.

MEYROWITZ J., 1985, No sense of place : the impact of electronic media on social behavior, New York, Oxford University Press.

MILLERAND F., S. PROULX et J. RUEFF (dir), 2010, Web social, mutation de la communication, Québec, Presses de l'Université du Québec.

MORNINGSTAR C. et F. R. FARMER, 1991, "The Lessons of Lucasfilm's Habitat », dans M. BENEDIKT (dir), Cyberspace: First Steps, Cambridge, MIT Press, pp.273-301.

O'REILLY T., 2005, "What Is Web 2.0. Design Patterns and Business Models for the Next Generation of Software ",. <http://oreilly.com/web2/archive/what-is-web-20.html>, dernière consultation le 4 mars 2010.

OIKARINEN J., 2004, "Foreword", dans P. MUTTON (dir), IRC Hacks, Sebastopol, O'Reilly Media, pp.vii-viii.

ONG W. J., 1982, Orality and literacy: the technologizing of the word, London; New York, Methuen.

PAQUIN L.-C., 2006, Comprendre les médias interactifs, Montréal, Isabelle Quentin éditeur. 
PASTINELLI M., 2007, Des souris, des hommes et des femmes au village global : parole, pratiques identitaires et lien social dans un espace de bavardage électronique, Québec, Presses de l'Université Laval.

PASTINELLI M., 2003, "La danse en ligne dans les voice chat du réseau Paltalk : une nouvelle modalité de l'être-ensemble dans l'espace virtuel », Colloque "Territoires partagés », ACFAS, 20 mai 2003, Université du Québec à Rimouski, <http://www.vrm.ca/documents/Pastinelli.pdf> (Résumé), dernière consultation le 3 mars 2010

PÉNARD T. et N. POUSSING, 2006, Usage d'Internet et investissement en capital social, Cahier de recherche no. 6, M@rsouin (Môle Armoricain de Recherche sur la Société de l'Information et les Usages d'INternet), <http://www. marsouin.org/IMG/pdf/Penard-Poussing_6-2006.pdf>, dernière consultation le 4 mars 2010.

PIEROZAK I., 2003, «Le français tchaté. Une étude en trois dimensions sociolinguistique, syntaxique et graphique - d'usages IRC », thèse de doctorat en linguistique, Université d'Aix-Marseille I, <http://www.upicardie.fr/LESCLaP/spip.php? rubrique43>, dernière consultation le 3 mars 2010.

PROULX S., 2009, "L'usage contributif: la production de contenus par des usagers au fondement du capitalisme informationnel », conférence d'ouverture au colloque "Contribuer dans l'univers Internet: le lien social au service de la production? ", 6 novembre 2009, Université du Québec à Montréal.

PROULX S. et M. SÉNÉCAL, 1995, "L'interactivité technique, simulacre d'interaction sociale et de démocratie? », Technologies de l'information et société, vol.7, $\mathrm{n}^{\circ} 2$, pp.239-255.

QUAN-HAASE A., 2008, "Instant Messaging on Campus: Use and Integration in University Students' Everyday Com-munication », The Information Society, vol.24, n², pp.105-115.

QUAN-HAASE A. et J. L. COLLINS, 2008, «'I'M THERE, BUT I MIGHT NOT WANT TO TALK TO YOU' ", Information, Communication \& Society, vol.11, $\mathrm{n}^{\circ} 4$, pp.526-543.

REID E., 1991, " Electropolis : Communication and Community on Internet Relay Chat ». thèse d'honneur de $1^{\text {er }}$ cycle, Département d'histoire, Université de Melbourne, <http://www.irchelp.org/irchelp/misc/electropolis.html>, dernière consultation le 4 mars 2010. 
Le chat est-il (encore) un média interactif ?

RINTEL S. et J. PITTAM, 1997, "Strangers in a Strange Land. Interaction Management on Internet Relay Chat», Human Communication Research, vol.23, ${ }^{\circ} 4$, pp.507-534.

SÉNÉCAL M., 2006, "Interactivité et interaction: sens, usages et pratiques », dans S. PROULX, L. POISSANT et M. SÉNÉCAL (dir), Communautés virtuelles: penser et agir en réseau, Québec, Presses de I'Université Laval, pp.133-164.

SHORT J., E. WILLIAMS et B. CHRISTIE, 1976, The Social Psychology of Telecommunications, New York, John Wiley \& Sons.

SULLIVAN B., 2005, "Yahoo chat choice signals Internet shift ». MSNBC, 23/06/2005, $<$ http://web.archive.org/web/2005072108 0722/www.msnbc.msn.com/id/8336384/>, dernière consultation le 28 janvier 2010.

SUSSAN R., 2010, "Les 7 bifurcations de la Réalité virtuelle (3/3): Hommes, machines et jeux", InternetActu, $<$ http://www.internetactu.net/2010/01/19/les-7-bifurcations-de-la-realite-virtuelle33-hommes-machines-et-jeux/>, dernière consultation le 27 janvier 2010.

UNDERNET USER COMMITTEE, 2003, « Undernet Anniversary » (verbatim d'une session de chat tenue le 8 février 2003 à l'occasion du 10e anniversaire d'Undernet), <http://www.user-com.undernet.org/promotions/anniversary.php>, dernière consultation le 28 février 2010.

VALLEE J., R. JOHANSEN, H. LIPINSKI, K. SPANGLER, T. WILSON et A. HARDY, 1975, Group Communication Through Computers. Volume 3: Pragmatics and Dynamics. Menlo Park, CA, Institute for the Future, $<$ http://eric.ed.gov/ERICDocs/data/ ericdocs2sql/content_storage_01/0000019b/80/38/c6/b3.pdf>, dernière consultation le 3 mars 2010.

VALLEE J., H. M. LIPINSKI et R. H. MILLER, 1974, Group Communication Through Computers. Volume 1: Design and Use of the FORUM System. Menlo Park, CA, Institute for the Future,<http://eric.ed.gov/ERICDocs/data/ericdocs2sql/content_storage_01/000 0019b/80/36/be/89.pdf>, dernière consultation le 3 mars 2010.

WATT S. E., M. LEA et R. SPEARS, 2002, "How Social is Internet Communication? A Reappraisal of Bandwidth and Anonymity Effects », dans S. WOOLGAR (dir), Virtual Society? Technology, Cyberbole, Reality, Oxford, Oxford University Press, pp.61-77. 
WENGER E., 2000, "Communities of Practice and Social Learning Systems », Organization, vol.7, n², pp.225-246.

WOOLLEY D. R., 1994, " PLATO: The Emergence of Online Community », <http://www.thinkofit.com/plato/dwplato.htm>, dernière consultation le 4 mars 2010.

YAKAL K., 1986, "Habitat. A Look At The Future Of Online Games », COMPUTE!, $\quad \mathrm{n}^{\circ} 77, \quad$ pp.32, <http://www. atarimagazines.com/compute/issue $77 /$ habitat.php $>$, dernière consultation le 20 novembre 2009. 